\title{
Revealing the local atomic and magnetic structure of a new dilute ferromagnetic semiconductor by pair distribution function analysis
}

\author{
Benjamin A. Frandsen \\ Department of Physics, University of California, Berkeley
}

Dilute ferromagnetic semiconductors (DFSs) are materials with semiconducting transport behavior that have been doped with a small amount of magnetic impurities to induce ferromagnetism. They hold the promise of novel functionality and improved performance in "spintronic" devices, but they have been challenging to study experimentally because prototypical DFSs such as (Ga,Mn)As are notoriously difficult to synthesize, stabilizing only in thin-film form. Recently, a promising new class of DFS materials based on the chemistry and structure of iron-based superconductors has been discovered, including the compound $(\mathrm{Ba}, \mathrm{K})(\mathrm{Zn}, \mathrm{Mn})_{2} \mathrm{As}_{2}$. These materials have similar magnetic ordering temperatures as state-ofthe-art (Ga,Mn)As and can be synthesized in bulk form, providing an unprecedented opportunity to study the mechanism of ferromagnetism in semiconductors. We have conducted a detailed temperature-dependent characterization of the atomic and magnetic structure of

$(\mathrm{Ba}, \mathrm{K})(\mathrm{Zn}, \mathrm{Mn})_{2} \mathrm{As}_{2}$ using atomic and magnetic pair distribution function analysis. The results establish the existence of an unexpected structural distortion on a short length scale of $\sim 5 \AA$, confirm long-range ferromagnetic order with the Mn spins aligned along the crystallographic $c$ axis, and reveal surprisingly robust short-range ferromagnetic correlations that persist even at room temperature. These findings fill in important gaps in the experimental understanding of DFS materials and provide guidance to their theoretical description. 